3. Grace E. Bates, Free loops and nets and their generalizations, Amer. J. Math. vol. 69 (1947) pp. 499-550.

4. R. H. Bruck, An extension theory for a certain class of loops, Bull. Amer. Math. Soc. vol. 57 (1951) pp. 11-26.

5. - Loops with transitive automorphism groups, Pacific Journal of Mathematics vol. 1 (1951) pp. 481-483.

6. P. T. Bateman, A remark on infinite groups, Amer. Math. Monthly vol. 57 (1950) pp. 623-624.

7. Raphael Artzy, On loops with a special property, to appear in Proc. Amer. Math. Soc.

UNIVERSITY OF WisCONSIN

\title{
THE SCHWARZIAN DERIVATIVE AND CONVEX FUNCTIONS
}

\section{RICHARD F. GABRIEL}

1. Introduction. In a comparatively recent paper [2], Nehari has shown that if

$$
f(z)=1 / z+a_{1} z+a_{2} z^{2}+\cdots \quad \text { for } 0<|z|<1
$$

and

$$
|\{f(z) ; z\}| \leqq \frac{\pi^{2}}{2}
$$$$
\text { for }|z|<1 \text {, }
$$

where $\{f(z), z\}$ is the Schwarzian derivative of $f(z)$ with respect to $z$, then $f(z)$ is univalent in the unit circle. The methods of Nehari can be modified to apply to functions of the form (1.1) to be shown univalent and convex in the unit circle. The principal result obtained in this paper is the following:

ThEOREM 1. If $f(z)$ is of the form (1.1), regular for $0<|z|<1$, and if

$$
|\{f(z), z\}| \leqq 2 c_{0}
$$

$$
\text { for }|z|<1 \text {, }
$$

where $c_{0}$ is the smallest positive root of the equation

$$
2 x^{1 / 2}-\tan x^{1 / 2}=0,
$$

then $f(z)$ is univalent in $0<|z|<1$ and maps the interior of each circle $|z|=r<1$ onto the exterior of a convex region. The constant $c_{0}$ is the largest possible one.

Presented to the Society, April 24, 1954; received by the editors May 20, 1954. 
2. Introductory topics. Let the function $p(z)$ be analytic and singlevalued for $|z|<1$. The differential equation

$$
w^{\prime \prime}+p(z) w=0
$$

will have two linearly independent solutions $w_{1}(z)$ and $w_{2}(z)$ which are uniquely determined by the conditions

$$
\begin{array}{ll}
w_{1}(0)=1, & w_{1}^{\prime}(0)=0, \\
w_{2}(0)=0, & w_{2}^{\prime}(0)=1 .
\end{array}
$$

These solutions are analytic and single-valued for $|z|<1$ and consequently have the following power series expansions valid for $|z|<1$

$$
w_{1}(z)=1+\sum_{n=2}^{\infty} a_{n} z^{n}, \quad w_{2}(z)=z+\sum_{n=2}^{\infty} b_{n} z^{n} .
$$

For these solutions the Wronskian, $W\left(w_{1}, w_{2}\right)$, is

$$
W\left(w_{1}, w_{2}\right)=\left|\begin{array}{cc}
w_{1}(z) & w_{2}(z) \\
w_{1}^{\prime}(z) & w_{2}^{\prime}(z)
\end{array}\right| \equiv 1 \quad \text { for }|z|<1 .
$$

It is known that for a function $f(z)$ of the form (1.1) to map the interior of each circle $|z|=r<1$ onto the exterior of a convex region, a necessary and sufficient condition is that

$$
\Re\left(1+\frac{z f^{\prime \prime}(z)}{f^{\prime}(z)}\right) \leqq 0 \quad \text { for }|z|<1 .
$$

Let $w_{1}(z)$ and $w_{2}(z)$ be the two linearly independent solutions of $(2.1)$ subject to the conditions (2.2). Consider

$$
f(z)=\frac{w_{1}(z)}{w_{2}(z)}=\frac{1}{z}+\cdots .
$$

Then

$$
\begin{aligned}
f^{\prime}(z)=\frac{w_{2} w_{1}^{\prime}-w_{2}^{\prime} w_{1}}{w_{2}^{2}(z)} & =-\frac{W\left(w_{1}, w_{2}\right)}{w_{2}^{2}(z)}=-\frac{1}{w_{2}^{2}(z)}, \\
f^{\prime \prime}(z) & =\frac{2 w_{2}^{\prime}(z)}{w_{2}^{3}(z)} .
\end{aligned}
$$

With these substitutions in (2.5) we have

$$
\Re\left(1+\frac{z f^{\prime \prime}(z)}{f^{\prime}(z)}\right) \equiv 1-2 \Re\left(\frac{z w_{2}^{\prime}(z)}{w_{2}(z)}\right) .
$$


But condition (2.5) implies

$$
\Re\left(\frac{z w_{2}^{\prime}(z)}{w_{2}(z)}\right) \geqq 1 / 2 \quad \text { for }|z|<1
$$

and conversely.

The inequality $(2.10)$ indicates that $w_{2}(z)$ is starlike with respect to the origin in $|z|<1$. Since $w_{2}^{\prime}(0) \neq 0$ it follows that $w_{2}(z)$ is univalent for $|z|<1$. Thus, because $w_{2}(0)=0$, it follows that $w_{2}(z) \neq 0$ for $0<|z|$ $<1$ and $f(z)$ defined in (2.6) is analytic and single-valued in $0<|z|<1$ and has a simple pole at the origin.

The preceding remarks may be summarized as

TheOREM 2. The function $f(z)$ defined in (2.6) will be univalent and convex for $0<|z|<1$ if and only if $w_{2}(z)$ satisfies (2.10).

It is surprising to observe from (2.10) that the convexity of $f(z)$ depends only on $w_{2}(z)$. It has already been noted that $w_{2}(z)$ is starlike, but (2.10) indicates that $w_{2}(z)$ must be a member of a special class of starlike functions. An investigation of the geometric significance of this class of functions should prove of interest.

In the proof of Theorem 1, we shall make use of some relationships between the Schwarzian derivative $\{f(z), z\}$ and the solutions of the linear second order differential equation (2.1). Although these relations are, perhaps, generally known, they will be listed for convenient reference.

If $y_{1}(z)$ and $y_{2}(z)$ are any two linearly independent solutions of (2.1), the ratio

$$
y_{1}(z) / y_{2}(z)
$$

satisfies the differential equation

$$
\{f(z), z\}=2 p(z)
$$

where

$$
\{f(z), z\}=\left(\frac{f^{\prime \prime}}{f^{\prime}}\right)^{\prime}-\frac{1}{2}\left(\frac{f^{\prime \prime}}{f^{\prime}}\right)^{2} .
$$

Conversely any solution of (2.11) may be written as the ratio of two linearly independent solutions of (2.1). For if $w_{1}(z)$ and $w_{2}(z)$ are the two linearly independent solutions of (2.1) satisfying (2.2), then

$$
\begin{aligned}
& y_{1}(z)=c_{11} w_{1}(z)+c_{12} w_{2}(z), \\
& y_{2}(z)=c_{21} w_{1}(z)+c_{22} w_{2}(z),
\end{aligned}
$$


with

$$
\left|\begin{array}{ll}
c_{11} & c_{12} \\
c_{21} & c_{22}
\end{array}\right| \neq 0
$$

and the ratio

$$
\frac{y_{1}(z)}{y_{2}(z)}=\frac{c_{11} w_{1}(z)+c_{12} w_{2}(z)}{c_{21} w_{1}(z)+c_{22} w_{2}(z)}
$$

involves three arbitrary constants. But the differential equation (2.11) is of third order. Hence (2.15) is a general solution and any solution of (2.11) may be obtained from (2.15) by a proper choice of constants. If $f(z)$ is to have the form (1.1), $c_{12}=c_{21}=0$ and $c_{11}=c_{22}=1$. From this it follows that

$$
f(z)=w_{1}(z) / w_{2}(z) .
$$

Thus there will be no loss of generality if we confine our attention to the two particular solutions.

3. The function $c^{1 / 2} \cot \left(c^{1 / 2} z\right)$. Let $c_{0}$ be the smallest positive root of the equation (1.4). $c_{0}$ satisfies the inequalities

$$
\begin{aligned}
\pi / 3<1.16 & <c_{0}^{1 / 2}<1.17<\pi / 2, \\
c_{0} & <\pi^{2} / 4 .
\end{aligned}
$$

The differential equation

$$
w^{\prime \prime}+c w=0,
$$

has the solutions

$$
\begin{aligned}
& w_{1}(z)=\cos \left(c^{1 / 2} z\right)=1+\cdots, \\
& w_{2}(z)=c^{-1 / 2} \sin \left(c^{1 / 2} z\right)=z+\cdots,
\end{aligned} \quad|z|<1,
$$

which satisfy conditions (2.2).

LEMMA 3.1. Let $c_{0}$ be the smallest positive root of the equation (1.4). Let $f(z)=c^{1 / 2} \cot \left(c^{1 / 2} z\right), 0<c \leqq c_{0}$. Then $f(z)$ is univalent in $0<|z|<1$ and maps the interior of the unit circle onto the exterior of a convex region. The constant $c_{0}$ is the best possible in the sense that for $c>c_{0}$ there is a point $z_{0}$, with $\left|z_{0}\right|<1$, for which

$$
\Re\left(1+z_{0} \frac{f^{\prime \prime}\left(z_{0}\right)}{f^{\prime}\left(z_{0}\right)}\right)>0 .
$$

As noted in the introduction, if $f(z)$ is of the form (1.1) and satisfies 
(1.2), then $f(z)$ is univalent for $0<|z|<1$. The function $f(z)$ of the lemma is the ratio $w_{1}(z) / w_{2}(z)$ of the solutions (3.4) of the differential equation (3.3). Hence from (2.11)

$$
\{f(z), z\}=2 c \leqq 2 c_{0} \quad \text { for }|z|<1,
$$

and from (3.2) and (1.2) we conclude that $f(z)$ is univalent for $|z|<1$.

To establish the convexity of $f(z)$ it will suffice to prove that

$$
\Re\left(\frac{z w_{2}^{\prime}(z)}{w_{2}(z)}\right) \equiv \Re\left(\frac{c^{1 / 2} z \cos \left(c^{1 / 2} z\right)}{\sin \left(c^{1 / 2} z\right)}\right) \geqq 1 / 2
$$

for $|z|<1$ and $0<c \leqq c_{0}$. If we substitute $z=x+i y$ in (3.6), simplify, and rearrange terms, we obtain

$$
\begin{aligned}
\sin \left(c^{1 / 2} x\right) \cos \left(c^{1 / 2} x\right)\left[2 c^{1 / 2} x-\tan \left(c^{1 / 2} x\right)\right] \\
\geqq \sinh \left(c^{1 / 2} y\right) \cosh \left(c^{1 / 2} y\right)\left[\tanh \left(c^{1 / 2} y\right)-2 c^{1 / 2} y\right]
\end{aligned}
$$

for $0<c \leqq c_{0}$ and $x^{2}+y^{2} \leqq 1$. It will be noted that equality holds in (3.7) for $x= \pm 1, y=0$, and $c=c_{0}$. For $x^{2}+y^{2}<1, x \geqq 0, y \geqq 0$, and $0<c \leqq c_{0}$, the left-hand member of (3.7) is positive while the righthand member is either negative or zero. Thus since the map of $|z|=1$ is symmetric about the real and imaginary axes, (3.6) is established and the expression $\Re\left(z w_{2}^{\prime}(z) / w_{2}(z)\right)$ actually attains the value $1 / 2$ for $x= \pm 1, y=0$, and $c=c_{0}$. For $c=c_{0}+\epsilon, \epsilon>0$ and $\epsilon$ arbitrarily small, $\Re\left(z w_{2}^{\prime}(z) / w_{2}(z)\right)<1 / 2$.

4. Proof of Theorem 1. To facilitate the proof of the theorem two lemmas will be introduced.

LemMa 4.1. If $w(z)$ satisfies (2.1) with $w(0)=0$ and $w^{\prime}(0)=1$, then for $0<r<1$

(4.1) $|w| 2 \Re\left(\frac{z w^{\prime}(z)}{w(z)}\right) \equiv r \int_{0}^{r}\left|w^{\prime}\right|^{2} d \rho-r \int_{0}^{r} \Re\left(z^{2} p(z)\right)_{|z|=\rho} \frac{|w|^{2}}{\rho^{2}} d \rho$ for $|z|<1$.

Let $C$ be a rectifiable curve lying entirely within the unit circle and joining the origin to any point $z$ within the unit circle. If (2.1) is multiplied by $[w(z)]^{*}$ (* denotes complex conjugate) and integrated along $C$, the identity known as Green's transform is obtained.

$$
\left[[w(z)]^{*} \cdot w^{\prime}(z)\right]_{0}^{z}-\int_{0}^{z}\left|w^{\prime}\right|^{2} d z^{*}+\int_{0}^{z} p(z)|w|^{2} d z \equiv 0,
$$

$|z|<1$. 
In particular let $C$ be the path from the origin to $z=r e^{i 0}$ along the ray $\theta=$ constant. If we multiply (4.2) by $z$ and take the real part we obtain (4.1).

This particular form (4.1) of Green's transform appears to have been first used in a recent paper by Robertson [3].

LEMMA 4.2. Let $y(\rho)$ and $y^{\prime}(\rho)$ be continuous real functions of $\rho$ for $0 \leqq \rho<1$. For small values of $\rho$ let $y(\rho)=O(\rho)$. Then

(4.3) $0 \leqq r \int_{0}^{r}\left[y^{\prime}(\rho)\right]^{2} d \rho-c r \int_{0}^{r} y^{2}(\rho) d \rho-c^{1 / 2} r \cot \left(c^{1 / 2} r\right) \cdot y^{2}(r)$

for $0<r<1$ and $c>0$. Equality holds for

$$
y(\rho)=c^{-1 / 2} \sin \left(c^{1 / 2} \rho\right),
$$

To prove this lemma, consider

$$
0 \leqq r \int_{0}^{r}\left[y^{\prime}(\rho)-c^{1 / 2} \cot \left(c^{1 / 2} \rho\right) \cdot y(\rho)\right]^{2} d \rho .
$$

Expanding, integrating the middle term by parts, and simplifying slightly, we obtain

$$
0 \leqq r \int_{0}^{r}\left[y^{\prime}(\rho)\right]^{2} d \rho-c^{1 / 2} r\left[\cot \left(c^{1 / 2} \rho\right) \cdot y^{2}(\rho)\right]_{0}^{r}-r c \int_{0}^{r} y^{2}(\rho) d \rho .
$$

With some further slight simplification the lemma follows.

Equality will hold in (4.3) if the integrand in (4.5) is zero. The resulting differential equation has (4.4) as a solution.

Theorem 1 now follows quite readily. The univalence of the function (1.1) is an immediate consequence of (1.3), (3.2), and the criterion of Nehari cited in the introduction.

From (2.11) and (1.3),

so that

$$
|p(z)| \leqq c_{0}
$$

$$
\Re\left(z^{2} p(z)\right) \leqq c_{0}|z|^{2}
$$$$
\text { for }|z|<1 \text {. }
$$

If $\Re\left(z^{2} p(z)\right)$ is replaced by this bound in (4.1) we obtain

$$
|w|^{2} \Re\left(\frac{z w}{w}\right) \geqq r \int_{0}^{r}\left|w^{\prime}\right|^{2} d \rho-r c_{0} \int_{0}^{r}|w|^{2} d \rho, \quad 0<r<1 .
$$

With $z=\rho e^{i \theta}$, let $w=u(\rho, \theta)+i v(\rho, \theta)$. Along the ray $\theta=$ constant, $w$ is a function of $\rho . u(\rho)$ satisfies the hypotheses of Lemma 4.2 as does $v(\rho)$. Substituting these functions in (4.3) we obtain the inequalities 


$$
\begin{aligned}
& 0 \leqq r \int_{0}^{r} u_{\rho}^{2} d \rho-c_{0} r \int_{0}^{r} u^{2} d \rho-c_{0}^{1 / 2} r \cot \left(c_{0}^{1 / 2} r\right) \cdot u^{2}(r, \theta), \\
& 0 \leqq r \int_{0}^{r} v_{\rho}^{2} d \rho-c_{0} r \int_{0}^{r} v^{2} d \rho-c_{0}^{1 / 2} r \cot \left(c_{0}^{1 / 2} r\right) v^{2}(r, \theta) .
\end{aligned}
$$

Adding these and simplifying we have

$$
\text { (4.10) } r \int_{0}^{r}\left|w^{\prime}\right|^{2} d \rho-c_{0} r \int_{0}^{r}|w|^{2} d \rho \geqq c_{0}^{1 / 2} r \cot \left(c_{0}^{1 / 2} r\right)|w|^{2} \text {. }
$$

Comparing (4.8) and (4.10) we see that

$$
\Re\left(\frac{z w}{w}\right) \geqq c_{0}^{1 / 2} r \cot \left(c_{0}^{1 / 2} r\right) \quad \text { for }|z|<1 .
$$

In Lemma 3.1 it was shown that $\Re\left(c^{1 / 2} z \cot \left(c^{1 / 2} z\right)\right) \geqq 1 / 2$ for $0<c \leqq c_{0}$ and $|z|<1$. The particular solution $w_{2}(z)$ satisfies the hypothesis of Lemma 4.1 and may be substituted for $w(z)$ in (4.11). We have finally

$$
\Re\left(\frac{z w_{2}^{\prime}(z)}{w_{2}(z)}\right) \geqq 1 / 2 \quad \text { for }|z|<1 .
$$

\section{Some consequences of Theorem 1.}

Corollary 5.1. Let $c_{0}$ be the smallest positive root of the equation (1.4). Let

$$
F(z)=z+b_{2} z^{2}+\cdots
$$

be analytic in $|z|<1$ with

$$
|\{F(z), z\}| \leqq 2 c_{0} \quad \text { for }|z|<1 .
$$

Then $F(z)$ maps the interior of the unit circle onto a region which is starlike with respect to the origin and every circle passing through the origin cuts the boundary of the region in, at most, two points.

For the function $f(z)=1 / F(z)$ we have

$$
\{f(z), z\}=\{F(z), z\} .
$$

From (5.2) and Theorem 1, $f(z)$ is convex. The boundary of the region mapped by $f(z)$ is cut by any straight line in, at most, two points. Under the transformation $F(z)=1 / f(z)$ the region mapped by $f(z)$ goes into the region mapped by $F(z)$ and straight lines transform into circles through the origin. Since $f(z)$ maps each circle $|z|=r<1$ onto the exterior of a convex region, and a fortiori a starlike region, 


$$
\Re\left(\frac{z f^{\prime}(z)}{f(z)}\right) \leqq 0
$$

for $|z|<1$.

But

$$
\Re\left(\frac{z F^{\prime}(z)}{F(z)}\right)=-\Re\left(\frac{z f^{\prime}(z)}{f(z)}\right) \geqq 0 \quad \text { for }|z|<1
$$

and it follows that $F(z)$ is starlike with respect to the origin for $|z|<1$.

THEOREM 3. Let

$$
g(z)=z+b_{2} z^{2}+\cdots
$$

be analytic for $|z|<1$ and real on the real axis. Let

$$
\Re\left(\left\{z g^{\prime}(z), z\right\}\right) \geqq-\frac{\pi^{2}}{2} \quad \text { for }|z|<1 .
$$

Then $g(z)$ is univalent in $|z|<1$ and maps the interior of the unit circle into a region which is convex in the direction of the imaginary axis. The constant $-\pi^{2} / 2$ is the best possible one.

Fejér [1] has shown that if $z g^{\prime}(z)$ is typically-real, then $g(z)$ is univalent and convex in the direction of the imaginary axis. The proof then reduces to showing that $z g^{\prime}(z)$ is typically-real. For convenience let

$$
h(z)=z g^{\prime}(z)=z+\cdots, \quad|z|<1 .
$$

It follows from the definition of $g(z)$ that $h(z)$ is real for real $z$. For any point $z_{0}$ with $\left|z_{0}\right|<1$

$$
h\left(z_{0}\right)=\left[h\left(z_{0}^{*}\right)\right]^{*} .
$$

If $h(z)$ is to satisfy the differential equation (2.11) then, as noted earlier, $h(z)=y_{1}(z) / y_{2}(z)$ where $y_{1}(z)$ and $y_{2}(z)$ are any two linearly independent solutions of (2.1). However, if $h(z)$ is to be of the form (5.8), the two solutions must be normalized and

$$
h(z)=\frac{w_{2}(z)}{w_{1}(z)}
$$

where $w_{1}(z)$ and $w_{2}(z)$ are the two solutions of (2.1) satisfying conditions (2.2).

If $h(z)$ is not typically-real there is a value $z_{1}$, with $\left|z_{1}\right|<1$ and $\Im\left(z_{1}\right) \neq 0$, and some real number $\alpha$ such that 


$$
h\left(z_{1}\right)=h\left(z_{1}^{*}\right)=\alpha .
$$

This is equivalent to saying that the function $w(z)$ where

$$
w(z)=w_{2}(z)-\alpha w_{1}(z)
$$

has zeros at $z_{1}$ and $z_{1}^{*}$. From this point the proof requires but a slight modification of that of Nehari's Theorem II in [2] and will not be repeated here.

The function $w(z)=e^{\pi z}$ shows that $-\pi^{2} / 2$ is the best possible constant, where $w(z)$ denotes $1+\pi z g^{\prime}(z)$.

\section{BIBLIOGRAPHY}

1. L. Fejêr, Neue Eigenschaften der Mittelwerte bei den Fourierreihen, J. London Math. Soc. vol. 8 (1933) p. 61, footnote.

2. Z. Nehari, The Schwarzian derivative and schlicht functions, Bull. Amer. Math. Soc. vol. 55 (1949) pp. 545-551.

3. M. S. Robertson, Schlicht solutions of $W^{\prime \prime}+p W=0$, Trans. Amer. Math. Soc. vol. 76 (1954) pp. 254-274.

RUTGERS UNIVERSITY 